\title{
Priorale Saint-Pierre de Souvigny (Allier), étude archéologique de la nef, première tranche
}

Pascale Chevalier, Morana Čaušević-Bully, Mathias Dupuis, Laurent Fiocchi et Olivier Lapie

\section{(2) OpenEdition}

\section{Édition électronique}

URL : https://journals.openedition.org/cem/1087

DOI : $10.4000 /$ cem. 1087

ISSN : 1954-3093

Éditeur

Centre d'études médiévales Saint-Germain d'Auxerre

Édition imprimée

Date de publication : 15 août 2007

ISSN : 1623-5770

Référence électronique

Pascale Chevalier, Morana Čaušević-Bully, Mathias Dupuis, Laurent Fiocchi et Olivier Lapie, « Priorale Saint-Pierre de Souvigny (Allier), étude archéologique de la nef, première tranche », Bulletin du centre d'études médiévales d'Auxerre | BUCEMA [En ligne], 11 | 2007, mis en ligne le 30 août 2007, consulté le 22 septembre 2022. URL : http://journals.openedition.org/cem/1087 ; DOI : https://doi.org/10.4000/ cem. 1087

Ce document a été généré automatiquement le 22 septembre 2022.

\section{(c)}

Creative Commons - Attribution - Pas d'Utilisation Commerciale - Partage dans les Mêmes Conditions 4.0 International - CC BY-NC-SA 4.0

https://creativecommons.org/licenses/by-nc-sa/4.0/ 


\title{
Priorale Saint-Pierre de Souvigny (Allier), étude archéologique de la nef, première tranche
}

\author{
Pascale Chevalier, Morana Čaušević-Bully, Mathias Dupuis, Laurent \\ Fiocchi et Olivier Lapie
}

1 En 2002, nous avions présenté d'abord dans le bulletin du CEM ${ }^{1}$ puis à Auxerre les découvertes effectuées en 2001 et 2002 autour du tombeau des saints Mayeul et Odilon de Cluny dans l'église Saint-Pierre de Souvigny (Allier). Nous rendons brièvement compte ici des travaux menés sur le même site en 2006 - travaux en partie limitrophes de la travée explorée précédemment (fig.1). La première tranche de l'étude archéologique envisagée s'inscrit dans le cadre d'une réfection intérieure des cinq vaisseaux de la nef de la grande priorale clunisienne, portée par la Conservation régionale des Monuments historiques d'Auvergne. L'intervention, motivée tant par l'intérêt scientifique du site que par les risques de destruction engendrés par la réfection, avait pour objectif d'étudier en 2006 les deux collatéraux nord; précédée par un sondage en avril, elle a débuté en juin et s'est achevée en novembre ${ }^{2}$. La topographie interne médiévale présentait un pendage ouest/est très marqué. En 1835, lors des travaux qui ont suivi le classement de l'édifice, tous les niveaux modernes et médiévaux ont été nivelés à l'ouest pour rétablir un sol plus horizontal ${ }^{3}$. Il ne subsiste à l'est que les premiers sols postérieurs au XII ${ }^{e}$ siècle. En outre, quelques piles de la nef ont été consolidées au béton armé au début du XXe siècle ; $25 \mathrm{~m}^{2}$ de surface est à chaque fois stérile alentour.

Cadre architectural de l'intervention

2 L'édifice actuel (87 m de long x $40 \mathrm{~m}$ de largeur maximum à l'est; largeur de la nef : $28 \mathrm{~m}$ ) résulte de plusieurs chantiers de construction qui se sont succédés entre le milieu $\mathrm{du} \mathrm{XI}{ }^{\mathrm{e}}$ et le XVIII ${ }^{\mathrm{e}}$ siècle. Existait, en outre, auparavant, un bâtiment dont on ignore l'implantation exacte: l'église Saint-Pierre de la donation à Cluny de sa villa de Sylviniacum par Aymar, l'ancêtre des Bourbons, en 915-920 4 . 
3 L'église charpentée construite en 1040-1060 comportait un chevet tri-absidé ouvrant sur un chœur rectangulaire à alvéoles latérales inscrites, un transept et une nef assez large, dont la première tranche de l'étude n'a atteint que le tiers septentrional. Dans la phase suivante, la nef fut divisée en 3 vaisseaux; nous en avons exploré le bas-côté nord en 2006. Au sud, s'étendaient les bâtiments claustraux ordonnés autour du cloître et, au sud-est, selon un système très clunisien, une chapelle dédiée à la Vierge Marie ${ }^{5}$. À la fin du XI ${ }^{\mathrm{e}}$ siècle, une galilée fut édifiée à l'ouest des deux tours de façade, à peine terminées. Le développement rapide du pèlerinage et de la communauté amena une refonte de la priorale et des bâtiments monastiques dans le courant du XII ${ }^{\mathrm{e}}$ siècle. On ajouta un large bas-côté sur chaque flanc de la nef, en perçant des arcades dans les anciens murs gouttereaux, pour former une nef à cinq vaisseaux voûtés (dont nous avons pu étudier en 2006 les deux collatéraux nord). Cette campagne entraîna un remodelage du cloître et des bâtiments qui flanquaient jusque-là le bas-côté sud. Puis, on agrandit l'église vers l'est en construisant vers 1150-1165 au-delà d'un second transept, comme à Cluny III, un chevet à déambulatoire et cinq chapelles rayonnantes. Enfin, dom Geoffroy Chollet consacra les 30 ans de son priorat (1424-1454) à une réfection d'ensemble ${ }^{6}$, voûtant sur croisées d'ogives le haut vaisseau de la nef et les transepts, reconstruisant le chœur, faisant abattre la galilée et construire devant les tours romanes la façade actuelle, etc. Les modifications suivantes ne touchèrent pas la nef. Église de pèlerinage auprès des saints Mayeul et Odilon, la priorale clunisienne était la nécropole dynastique des ducs de Bourbonnais, le « Saint-Denis des Bourbons ». Elle avait aussi un rôle paroissial, avec un autel Saint-Nicolas situé dans le bas-côté extérieur nord, (cf. infra).

Villa carolingienne (?) et premier horizon funéraire

4 Des vestiges de la villa carolingienne, connue par la charte de donation à cluny (915-920), sont apparus, mais leur profondeur d'enfouissement rend l'examen ardu vers l'est : d'abord un fossé orienté nord-sud (L. obs. 5,08 m) excavé dans le substrat glaiseux et un trou de poteau sur sa rive orientale. Le comblement progressif du fossé contenait une quantité notable d'ossements d'animaux et de tessons de céramique, datable des $\mathrm{VIII}^{\mathrm{e}}-\mathrm{X}^{\mathrm{e}}$ siècles. Environ $10 \mathrm{~m}$ plus à l'est, un trou de poteau à calage de pierres et le sol d'une cour (?), mis au jour sur 4,50 $\mathrm{m}^{2}$, associé à un petit trou de poteau et au départ d'une sablière orientée nord-sud ressortent de la même période. On a enfin observé à l'est de la zone étudiée un tronçon (L. 4,70 m) d'un mur en petit appareil (ép. $74 \mathrm{~cm}$ ), orienté nord-est/sud-ouest, qui est emporté par le gouttereau nord primitif de la priorale romane (mi-XI ${ }^{\mathrm{e}}$ siècle), orientée quant à elle légèrement est-nord-est/ouestsud-ouest. Il est impossible de préciser la date, l'ampleur et la fonction de ce bâtiment ancien.

5 Huit sépultures en cercueil monoxyle ${ }^{7}$ orientées ouest-est représentent l'horizon ultérieur; deux exemplaires ayant conservé leur couvercle recoupent le fossé carolingien comblé. Ces cercueils ont été fabriqués dans des grumes de chênes d'environ 250 ans à l'abattage, datés par dendrochronologie des années $940^{8}$; quatre d'entre eux ont été fouillés, avec de forts déplacements des ossements par l'eau qui a favorisé la conservation du bois. On aurait là des individus adultes, d'une catégorie aisée de la population de l'ancienne villa (?), inhumés juste avant l'installation des moines en 954 ou au tout début du monastère ${ }^{9}$. Ces contenants monoxyles paraissent remplacer dans la région les sarcophages de grès de la période précédente. Notons encore trois sépultures d'enfants et une d'adulte. Un sol de glaise couvert d'un radier 
de pierres et un lit de mortier, qui constituent le sol au nord de l'église à la phase suivante, passent au-dessus de ces tombes du $\mathrm{X}^{\mathrm{e}}$ siècle, peut-être concomitamment avec une structure ligneuse (plancher?), mise au jour à l'ouest sur 2,62 x 0,96 m, dont les planches suivent l'orientation nord/sud du fossé.

Nef du XI ${ }^{e}$ siècle et cimetière extérieur

6 L'édifice du milieu du XI ${ }^{\mathrm{e}}$ siècle est mononef. Un mur de refend nord/sud, dont l'existence n'était pas soupçonnée auparavant, a été attesté sur $1,76 \mathrm{~m}$ de longueur et trois assises d'élévation. De même épaisseur que le gouttereau nord $(90 \mathrm{~cm})$, il fermerait une avant-nef originelle, ayant la profondeur de deux des futures travées de la fin du XI ${ }^{e}$ siècle ; elle est supprimée dès la phase suivante. La nef, dont le premier sol est établi sur une semelle d'isolation et de stabilisation ou sur un remblai de matériaux de construction, est rapidement soumise à un violent incendie, puis semble avoir été brièvement restaurée, mais le sinistre a probablement influé sur la décision de modifier le bâtiment dans le dernier tiers ou quart du XI ${ }^{\mathrm{e}}$ siècle. La nef est alors subdivisée en trois vaisseaux par deux files de piles quadrilobées. Les bas-côtés étroits $(2,70 \mathrm{~m}$ au nord) reçoivent des berceaux sur doubleaux retombant sur des colonnes engagées dans les gouttereaux. On a pu observer les fondations et la mouluration des bases de cinq des piles nord à plinthe cruciforme, édifiées en moyen appareil de grès sur des fondations en massif quadrangulaire. Le parement sud du gouttereau est retravaillé à chaque travée pour insérer des colonnes engagées, en moyen appareil de grès. On rétrécit aussi les larges fenêtres du premier état. Les sols (lits de mortier alternant avec de la terre indurée) se succèdent rapidement, avec le négatif rectangulaire d'un autel éphémère à mi-longueur du bas-côté. L'humidité ambiante constitue un problème récurrent dans l'église, fondée dans la glaise : un petit caniveau à ciel ouvert file d'ouest en est dans le bas-côté. Un muret en moyen appareil, déjà observé en 2002, isole le collatéral du vaisseau central dans la travée qui abrite la tombe des saints Mayeul et Odilon ${ }^{10}$. Comme le caniveau et l'autel, il est abandonné et nivelé au début du XII ${ }^{\mathrm{e}}$ siècle.

7 Quelques sépultures appartiennent au cimetière paroissial extérieur du $\mathrm{XI}^{\mathrm{e}}$ siècle. Orientées parallèlement au gouttereau nord (est-nord-est/ouest-sud-ouest) et non plus strictement ouest-est comme les précédentes, ces inhumations d'adultes en pleine terre ou en cordons de pierres, étaient très rapprochées et pour certaines se chevauchaient en partie. La cuve d'un sarcophage trapézoïdal en grès, datable des $\mathrm{VI}^{\mathrm{e}}-\mathrm{VII}{ }^{\mathrm{e}}$ siècles, semble avoir été remployée sans couvercle durant cette phase.

Ajout du bas-côté paroissial au XII ${ }^{e}$ siècle et inhumations du bas Moyen Âge à l'époque moderne

8 La nouvelle avant-nef (galilée) doit être à peine terminée, quand vers 1110 - pour agrandir la nef d'un bas-côté externe - on perce six grandes arcades dans le gouttereau nord du XI siècle. Six piles irrégulières, quadrilobées autour d'un massif trapézoïdal, sont créées à partir de tronçons du gouttereau du $\mathrm{XI}^{\mathrm{e}}$ siècle. Le tout est accompagné de reprises des fondation, d'un terrassement et d'un rehaussement général du sol. L'empreinte du massif rectangulaire maçonné de l'autel Saint-Nicolas subsiste à l'extrémité est du nouveau bas-côté paroissial nord; dans un second temps, on insère dans ses angles les quatre colonnettes d'un ciborium.

9 Après un hiatus de plus de 250 ans, le troisième horizon funéraire (fin du XIV $\mathrm{XVIII}^{\mathrm{e}}$ siècle) occupe inégalement les six travées de la nef nord (paroissiale). Une quinzaine de sépultures en cercueils cloutés ou chevillés, en coffres ou en pleine terre a été examinée, ainsi qu'un caveau avec système de pourrissoir, contenant au moins dix- 
sept individus adultes, sous un enfeu gothique. Un drain, fait d'une planche de bois entre deux rangées de blocs de remploi, couvertes par des dalles, évacuait les eaux de ruissellement vers le cloitre (au XVI ${ }^{e}$ siècle ?) ; il s'est bouché par calcification.

Une tombe de prestige ad sanctos à la fin du XIV e siècle : celle du «bâtard » de Bourbon

Enfin, un sondage a été mené au sud pour identifier le tombeau, connu par un dessin ${ }^{11}$

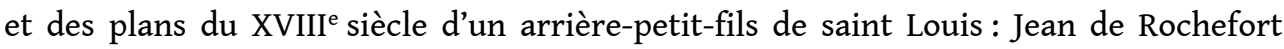
(+1375), fils naturel de Louis $\mathrm{I}^{\mathrm{er}} \mathrm{de}$ Bourbon. Un socle quadrangulaire, support du monument gothique disparu à deux gisants, coiffe l'extrados de la voûte d'un caveau, grossièrement obturé à l'ouest à la fin du XIV ${ }^{e}$ siècle par un muret en blocs de remploi romans. L'examen est resté superficiel, mais il apparaît qu'un premier individu (Jean de Rochefort ?) est inhumé dans une fosse rectangulaire de la taille du caveau; sur les bords de cette fosse on élève les parois qui soutiennent une voûte en berceau très inégal. Un deuxième corps est placé au-dessus (celui de son épouse dame Agnès Chalheu, après le 15 octobre 1389, date de son testament ?). La fosse d'accès ouest trop étroite n'a pas permis d'éviter que l'introduction en force du second corps ne repousse vers l'est une partie du squelette de la sépulture primaire. Deux autres tombes côtoyaient ce caveau dans ce qui devait être une chapelle funéraire.

L'ensemble des données collectées sera étudié en 2007, parallèlement à une nouvelle campagne qui s'étendra d'avril à juillet 2007 au reste de la nef: collatéraux méridionaux et haut vaisseau - avec le complément de la fouille de 2001-2002 autour de la tombe de Mayeul et Odilon, la poursuite de l'enquête sur les phases les plus anciennes d'occupation du site, la mise au jour des chapelles privées gothiques dans les bas-côtés sud, mais aussi de la galerie nord du premier cloître roman et d'un segment contigu des bâtiments claustraux contemporains, etc.

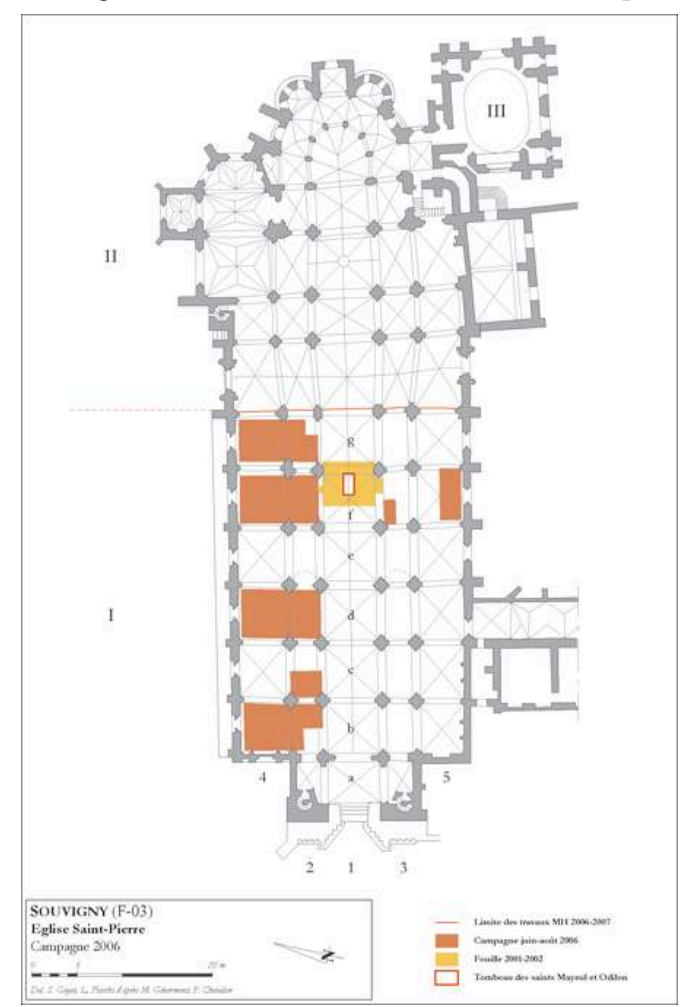

Priorale Saint-Pierre de Souvigny, plan des zones étudiées en 2006 (del. L. Fiocchi, sur fond de plan de S. Guyot d'après M. Génermont). 


\section{NOTES DE FIN}

1. P. CheVAlier, A. MAQUet, A. PAillet, « Souvigny (Allier). La découverte du tombeau des saints Maïeul et Odilon de Cluny ", in Bulletin du Centre d'études médiévales, 6 (2001-2002), p. 28-32.

2. Financée par des crédits européens, le Ministère de la Culture, le contrat de plan État/Région Auvergne sur les édifices romans et le Conseil général de l'Allier, la campagne 2006 a employé de trois à quatre archéologues et un anthropologue pendant quatre mois pleins grâce à une gestion passant par ARTeHIS UMR 5594 du CNRS, P. Chevalier assurant la direction bénévole du chantier ; 75 personnes nous ont gracieusement prêté main forte, notamment une cinquantaine d'étudiants venus de toute la France et du Québec.

3. Il reste néanmoins une pente de quelque $37,5 \mathrm{~cm}$ (!) sur la longueur de la nef.

4. A. BERNARD et A. BRUEL, Chartes de Cluny, Paris, 1876-1903, t. I, p. 206, n² 217. Cf.

A. MAQUET, Cluny en Auvergne (910-1156), thèse dactylographiée de doctorat d'histoire, sous la dir. de M. Parisse, Université de Paris I, 2006, chap. 2.

5. P. CHEVAlIER, A. MAQUET, « Notre-Dame-des-Avents, la seconde église du prieuré de Souvigny ", in Nos Églises Bourbonnaises, 17 (2004), p. 51-65.

6. A. COURTILLÉ, Auvergne, Bourbonnais, Velay gothiques, Paris, 2002, p. 408-421.

7. Trois autres exemplaires ont été découverts à Souvigny même : un au début du XIX siècle (L.-J. ALARY, Petite géographie historique, commerciale, agricole et industrielle du département de l'Allier, Moulins, 1851, p. 255), un second par M. Génermont en 1935 (M. GÉNERMONT, « Les monuments historiques de l'Allier en 1935 », in Bulletin de la Société d'Émulation du Bourbonnais, 39 (1936), p. 271-272) et un $3^{\text {e }}$ daté par dendrochronologie des années 930-940, en 1991, dans la cour du prieuré du XVII ${ }^{\mathrm{e}}$ siècle (A.-M. JURQUET, "Souvigny (Allier). Prieuré », in Chronique des fouilles médiévales en France, Archéologie médiévale, 21 (1991), p. 334.).

8. Étude du CEDRE de Besançon.

9. Aymon, le fils d'Aymar, a tenté de remettre la main sur les biens donnés par son père, il les restitue par « déguerpissement » en 954 (Arch. dép. de l'Allier, série H 419 : Thesaurus Sylviniacensis). Cette date doit correspondre à l'installation réelle des moines clunisiens à Souvigny.

10. P. CHEVALIER, « Les tombeaux et les monuments funéraires médiévaux des saints abbés Mayeul et Odilon de Cluny ", in Hortus artium medievalium, 10 (2004), p. 119-132. 11. Paris, BnF, Clairambault 640, fol. 269 (dessin réalisé pour Roger de Gaignières). 